\title{
THE DECOMPOSITIONS OF SCHUR COMPLEXES
}

\author{
HYOUNG J. KO
}

\begin{abstract}
This paper presents a method for finding the characteristic-free Pieri type decompositions of Schur modules, Weyl modules, and Schur complexes. We also introduce several new combinatorial rules for computing the Littlewood-Richardson coefficients.
\end{abstract}

\section{INTRODUCTION}

The study of finite-free resolutions, modular representations, invariant theory, and algebraic geometry over fields of positive characteristics has led to the characteristic-free representation theory of the general linear group. Several authors constructed and investigated the representations of the general linear group over fields of arbitrary characteristics (see, for instance, [5]), but the question is far from being completely understood. Recently, it has been recognized that those constructions can be adequately adapted in order to make sense over arbitrary commutative rings; Akin, Buchsbaum, and Weyman [1, 2] have introduced and studied the Schur and Weyl functors which turn out to be the natural generalizations to commutative rings of the constructions of the classical representations of the general linear group given by I. Schur and H. Weyl, respectively. These functors are universally free and give rise to modules-Schur and Weyl modules-which are isomorphic over fields of characteristic zero, but are far from being isomorphic over the integers. Furthermore, Schur and Weyl modules are indecomposable, but they are not, in general, irreducible over commutative rings. Moreover, this important development admitted a natural generalization to Schur complexes, whose usefulness is abundant. For instance, Schur complexes play a central role in the resolutions of determinantal varieties $[2,8]$. This forces us to further study Schur complexes. One way to study Schur complexes is to look for complex-theoretic versions of classical character relations for the general linear group. We shall first specialize our questions in terms of modules. Let $F$ denote a finitely generated free module over a commutative ring $R$ with an identity. Then, the Schur module $L_{\lambda / \mu} F$ and the Weyl module $K_{\lambda / \mu} F$ are naturally $\mathrm{GL}(F)$-modules. If $R$ is a field of characteristic zero, we have the direct sum decompositions $L_{\lambda / \mu} F=\sum C_{\mu \nu}^{\lambda} L_{\nu} F$ and

Received by the editors March 6, 1989 .

1980 Mathematics Subject Classification (1985 Revision). Primary 05A17, 13D25, 20C30; Secondary $15 \mathrm{~A} 72$. 
$K_{\lambda / \mu} F=\sum C_{\mu \nu}^{\lambda} K_{\nu} F$ for suitable partitions $\nu$ of weight $|\lambda|-|\mu|$, and suitable coefficients $C_{\mu \nu}^{\lambda}[2,3]$. In general, the above decompositions hold only up to filtration because the direct sum decompositions are natural only in characteristic zero. To extend such decompositions to the more general case in which the ground field is replaced by an arbitrary commutative ring, we need to describe those partitions $\nu$ by means of a filtration natural in every characteristic, and the coefficients $C_{\mu \nu}^{\lambda}$ in an efficient way.

With this example in mind, we will now sketch the outline of this article.

$\S 2$ covers the basic definitions and some of the important properties utilized in the main body of the paper.

In $\S 3$, we prove the analog for Schur complexes of the Pieri formulas for symmetric functions (or Schubert cycles). More precisely, let $\phi: G \rightarrow F$ be any homomorphism of finitely generated free modules over a commutative ring $R$. Then we construct universal filtrations of the Schur complexes $\mathbf{L}_{\lambda /(p)} \phi$ and $\mathbf{L}_{\lambda /\left(1^{p}\right)} \phi$ whose associated graded complexes are the direct sums of Schur complexes of the type which results in the classical case. Specializing our results, we obtain the characteristic-free Pieri type decompositions of Schur modules, Weyl modules, and Schur complexes.

$\S 4$ provides efficient algorithms for computing features of the LittlewoodRichardson coefficients $C_{\mu \nu}^{\lambda}$.

\section{SCHUR COMPLEXES}

Shape matrices. In this section, we will review some of the basic facts and give some of the basic notation that will be used throughout. For proofs and details, we refer the reader to the papers $[1,2]$ of the bibliography.

A partition is a nonincreasing sequence $\lambda=\left(\lambda_{1}, \ldots, \lambda_{s}\right)$ of nonnegative integers $\lambda_{1} \geq \lambda_{2} \geq \cdots \geq \lambda_{s}$. We say that the number of its nonzero terms is its length, $l(\lambda)$. The weight of a partition $\lambda$ is the sum of the terms of $\lambda$ and is denoted by $|\lambda|$. We will denote by $\mathbf{N}^{\infty}$ the set of all infinite sequences of nonnegative integers containing only a finite number of nonzero terms. Given any finite sequence $\lambda=\left(\lambda_{1}, \ldots, \lambda_{s}\right)$ we may think of it as a sequence $\left(\lambda_{1}, \ldots, \lambda_{s}, 0, \ldots\right)$ in $\mathbf{N}^{\infty}$ by extension with zeros at the end. Thus, we will not distinguish between $\left(\lambda_{1}, \ldots, \lambda_{s}\right)$ and $\left(\lambda_{1}, \ldots, \lambda_{s}, 0, \ldots\right)$ in $\mathbf{N}^{\infty}$.

A relative sequence is a pair $(\lambda, \mu)$ of sequences in $\mathbf{N}^{\infty}$ such that $\mu \subseteq \lambda$ meaning that $\mu_{i} \leq \lambda_{i}$ for all $i$. We will use the notation $\lambda / \mu$ to represent relative sequences. If both $\lambda$ and $\mu$ are partitions, then the relative sequence $\lambda / \mu$ will be called a skew partition. It is natural to think of a sequence $\lambda$ in $\mathbf{N}^{\infty}$ as a relative sequence $\lambda /(0)$ by taking the zero sequence $(0)=(0,0, \ldots)$ as the second member of the pair.

Suppose now that $\lambda / \mu=\left(\lambda_{1}, \ldots, \lambda_{s}\right) /\left(\mu_{1}, \ldots, \mu_{s}\right)$ is a skew partition. The diagram of $\lambda / \mu$ is defined to be the set of all ordered pairs $(i, j)$ of integers satisfying the inequalities $1 \leq i \leq s, \mu_{i}+1 \leq j \leq \lambda_{i}$ simultaneously. The 
shape matrix of $\lambda / \mu$ is an $S \times \lambda_{1}$ matrix $A=\left(a_{i j}\right)$ defined by the rule

$$
a_{i j}= \begin{cases}1 & \text { if } \mu_{i}+1 \leq j \leq \lambda_{i} \\ 0 & \text { otherwise }\end{cases}
$$

As an example take $\lambda=(4,3,2)$ and $\mu=(2,1)$. Then the shape matrix of $\lambda / \mu$ is

and the diagram of $\lambda / \mu$ is

$$
\left(\begin{array}{llll}
0 & 0 & 1 & 1 \\
0 & 1 & 1 & 0 \\
1 & 1 & 0 & 0
\end{array}\right)
$$

where each dot represents an ordered pair $(i, j)$. It is often convenient to replace the dots by squares, in which case the diagram is

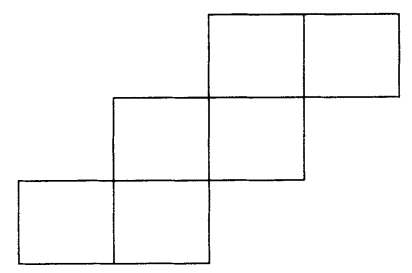

In general, we define a shape matrix to be a finite matrix with zeros and ones as entries. Given any relative sequence $\lambda / \mu$ we can associate to $\lambda / \mu$ a diagram and a shape matrix with the similar rules used for skew partitions. However, we will usually not distinguish between a relative sequence $\lambda / \mu$, its shape matrix $A=\left(a_{i j}\right)$ and its diagram $\Delta_{\lambda / \mu}$. The weight of a shape matrix $A=\left(a_{i j}\right)$ of a relative sequence $\lambda / \mu$ is defined to be $|\lambda|-|\mu|$ and is denoted by $|A|$, or simply $|\lambda / \mu|$.

If $\lambda=\left(\lambda_{1}, \lambda_{2}, \ldots\right) \in \mathbf{N}^{\infty}$ is a partition then its conjugate or transpose is defined to be the partition $\tilde{\lambda}=\left(\tilde{\lambda}_{1}, \tilde{\lambda}_{2}, \ldots\right)$ where $\tilde{\lambda}_{j}$ is the number of terms of $\lambda$ which are greater than or equal to $j$. Similarly, if $A=\left(a_{i j}\right)$ is a shape matrix we define the transpose $A^{t}:=\widetilde{A}=\left(\widetilde{a}_{i j}\right)$ of $A$ in the usual way by taking $\widetilde{a}_{i j}=a_{j i}$.

Let $A=\left(a_{i j}\right)$ be an $s \times t$ shape matrix. We define the row sequence $a_{A}=$ $\left(a_{1}, \ldots, a_{s}\right)$ of $A$ by $a_{i}=\sum_{j=1}^{t} a_{i j}$ for $i=1, \ldots, s$ and the column sequence $b_{A}$ to be $a_{\tilde{A}}$. Clearly $b_{A}=\left(b_{1}, \ldots, b_{t}\right)$ where $b_{j}=\sum_{i=1}^{s} a_{i j}$ for $j=1, \ldots, t$. That is, $a_{A}$ is just the sequence of row sums of $A$ and $b_{A}$ is the sequence of column sums of $A$.

Schur modules, Weyl modules, and Schur complexes. Let $R$ be a commutative ring and let $\phi: G \rightarrow F$ be any homomorphism of finitely generated free $R$ modules. We define the symmetric algebra $S \phi$ on the map $\phi$ to be the Hopf 
algebra $\Lambda G \otimes S F$ and the exterior algebra $\Lambda \phi$ on the map $\phi$ to be the Hopf algebra $D G \dot{\otimes} \Lambda F$, where $\dot{\otimes}$ denotes the 'twisted' tensor product over $R$. The element $C_{\phi} \in G^{*} \otimes F$ corresponding to the map $\phi \in \operatorname{Hom}_{R}(G, F)$ under the natural isomorphism $\operatorname{Hom}_{R}(G, F) \cong G^{*} \otimes F$ acts on both $S \phi$ and $\Lambda \phi$ making them into complexes. In particular, $C_{\phi}: \Lambda^{i} G \otimes S_{j} F \rightarrow \Lambda^{i-1} G \otimes S_{j+1} F$, where $C_{\phi} \in \Lambda G^{*} \otimes S F$, and $C_{\phi}: D_{i} G \otimes \Lambda^{j} F \rightarrow D_{i-1} G \otimes \Lambda^{j+1} F$, where $C_{\phi} \in S G^{*} \dot{\otimes} \Lambda F$.

Define $S_{p} \phi$ to be the complex

$$
0 \rightarrow \Lambda^{p} G \rightarrow \cdots \stackrel{C_{\phi}}{\rightarrow} \Lambda^{i} G \otimes S_{p-i} F \stackrel{C_{\phi}}{\rightarrow} \cdots \rightarrow G \otimes S_{p-1} F \rightarrow S_{p} F \rightarrow 0
$$

and define $\Lambda^{p} \phi$ to be the complex

$$
0 \rightarrow D_{p} G \rightarrow \cdots \stackrel{C_{\phi}}{\rightarrow} D_{i} G \otimes \Lambda^{p-i} F \stackrel{C_{\phi}}{\rightarrow} \cdots \rightarrow G \otimes \Lambda^{p-1} F \rightarrow \Lambda^{p} F \rightarrow 0 .
$$

It is clear that $S \phi=\sum_{p \geq 0} S_{p} \phi$ and $\Lambda \phi=\sum_{p \geq 0} \Lambda^{p} \phi$. It can also be checked that multiplication and comultiplication maps in $S \phi$ and $\Lambda \phi$ are compatible with the boundary maps. The reader should keep in mind that if $\phi$ is a zero map of $0 \rightarrow F$, then $S \phi$ is just the usual symmetric algebra $S F$ on $F$ and $\Lambda \phi$ is the exterior algebra $\Lambda F$ on $F$. Similarly, if $\phi$ is the map of $G \rightarrow 0$, then $S \phi$ is the exterior algebra $\Lambda G$ on $G$ and $\Lambda \phi$ is the divided power algebra $D G$ on $G$. Note that $S_{0} \phi=\Lambda^{0} \phi$ is the trivial complex $0 \rightarrow R \rightarrow 0$ and $S_{1} \phi=\Lambda^{1} \phi$ is $0 \rightarrow G \stackrel{\phi}{\rightarrow} F \rightarrow 0$.

Now we let $A$ be an $s \times t$ shape matrix and $a_{A}, b_{A}$ to be the sequences of row and column sums of $A$ as we did before. We can then define a map of chain complexes, called the Schur map, $d_{A}(\phi): \Lambda_{A} \phi \rightarrow S_{\tilde{A}} \phi$ to be the composition

$$
\begin{aligned}
\Lambda_{A} \phi & =\Lambda^{a_{A t}} \phi \rightarrow\left(\Lambda^{a_{11}} \phi \otimes \cdots \otimes \Lambda^{a_{1 t}} \phi\right) \otimes \cdots \otimes\left(\Lambda^{a_{s 1}} \phi \otimes \cdots \otimes \Lambda^{a_{s t}} \phi\right) \\
& \cong\left(\Lambda^{a_{11}} \phi \otimes \cdots \otimes \Lambda^{a_{s 1}} \phi\right) \otimes \cdots \otimes\left(\Lambda^{a_{1 t}} \phi \otimes \cdots \otimes \Lambda^{a_{s t}} \phi\right) \\
& \cong\left(S_{a_{11}} \phi \otimes \cdots \otimes S_{a_{s 1}} \phi\right) \otimes \cdots \otimes\left(S_{a_{1 t}} \phi \otimes \cdots \otimes S_{a_{s t}} \phi\right) \\
& \rightarrow S_{b_{1}} \phi \otimes \cdots \otimes S_{b_{t}} \phi=S_{b_{A}} \phi=S_{\tilde{A}} \phi,
\end{aligned}
$$

where the first map is diagonalization, the second is the isomorphism rearranging terms, the third is the isomorphism identifying $\Lambda^{a_{i j}} \phi$ with $S_{a_{i j}} \phi$ (for $a_{i j}=0$ or 1 ), and the last map is multiplication. (Notice that we use only formal properties of the Hopf algebras involved.) It should be observed that the Schur map $d_{A}(\phi)$ does not depend upon $\phi$, for only $G$ and $F$ are used. Since each of the maps comprising $d_{A}(\phi)$ is a map of complexes, the Schur map $d_{A}(\phi)$ is a natural transformation and a morphism of complexes. Hence, its image is a complex and we make the following definition.

Definition 2.1. The image of $d_{A}(\phi)$, denoted by $\mathbf{L}_{A} \phi$, is called the Schur complex on $\phi$ associated to the shape matrix $A$. When $A$ is the shape matrix of a skew partition $\lambda / \mu$, we write $\mathbf{L}_{\lambda / \mu} \phi$ instead of $\mathbf{L}_{A} \phi$. It should be noted that the Schur complexes $\mathbf{L}_{A} \phi$ are complexes of $\mathrm{GL}(G) \times \mathrm{GL}(F)$-modules (" $\mathrm{GL}(\phi)$ complexes", for short). 
Notice that $\mathbf{L}_{A}\left(\_\right)$is a functor from maps to complexes over $R$. If we restrict our attention to the maps of the form $\phi: 0 \rightarrow F$, then we write $d_{A}(F)$ for $d_{A}(\phi)$ and recover the usual Schur functor $L_{A} F$ on $R$-modules (in dimension zero) as the image of $d_{A}(F)$. When we view $L_{A} F$ as a $\mathrm{GL}(F)$-module in a natural way $L_{A} F$ will be referred to as the Schur module. Similarly, if we restrict attention to the maps of the form $\phi: G \rightarrow 0$ we write $d_{\dot{A}}(G)$ for $d_{A}(\phi)$ and obtain the Weyl (or Coschur) functor $K_{A} G$ (in dimension $|A|$ ) as the image of $d_{\dot{A}}(G)$. When viewed as a $\mathrm{GL}(G)$-module $K_{A} G$ will be referred to as the Weyl module.

Suppose now that $\phi: G \rightarrow F$ is the direct sum $\phi_{1} \oplus \phi_{2}$ of two maps $\phi_{i}: G_{i} \rightarrow F_{i}, i=1,2$. By this we mean that $G=G_{1} \oplus G_{2}, F=F_{1} \oplus F_{2}$, and $\phi\left(g_{1}, g_{2}\right)=\left(\phi_{1}\left(g_{1}\right), \phi_{2}\left(g_{2}\right)\right)$. For any nonnegative integer $p$ we have the direct sum decomposition

$$
\Lambda^{p} \phi=\sum_{a+b=p} \Lambda^{a} \phi_{1} \otimes \Lambda^{b} \phi_{2}
$$

of chain complexes. If $\mathbf{P}=\left(p_{1}, \ldots, p_{s}\right)$ is a sequence of nonnegative integers of length $s$, then (1) immediately yields a natural direct sum decomposition of the chain complexes $\Lambda_{\mathbf{P}} \phi=\Lambda^{p_{1}} \phi \otimes \cdots \otimes \Lambda^{p_{s}} \phi$ as follows

$$
\Lambda_{\mathbf{p}} \phi=\sum \Lambda_{\mathbf{a}} \phi_{1} \otimes \Lambda_{\mathbf{b}} \phi_{2},
$$

where the sum is taken over all sequences $\mathbf{a}=\left(a_{1}, \ldots, a_{s}\right), \mathbf{b}=\left(b_{1}, \ldots, b_{s}\right)$ of nonnegative integers such that $a_{i}+b_{i}=p_{i}$ for $i=1, \ldots, s$. Fix integers $a$ and $b$ such that $a+b=|\mathbf{P}|$, and define $\Lambda_{\mathbf{P}}\left(\phi_{1}, \phi_{2}: a, b\right)$ to be $\sum \Lambda_{\mathbf{a}} \phi_{1} \otimes \Lambda_{\mathbf{b}} \phi_{2}$ over all sequences $\mathbf{a}, \mathbf{b}$ of length $s$ satisfying $\mathbf{a}+\mathbf{b}=\mathbf{P},|\mathbf{a}|=a$, and $|\mathbf{b}|=b$. It follows from (2) that there is a direct sum decomposition

$$
\Lambda_{\mathbf{P}} \phi=\sum_{a+b=p} \Lambda_{\mathbf{P}}\left(\phi_{1}, \phi_{2} ; a, b\right)
$$

where $p=|\mathbf{P}|$. The above discussion and definitions may be repeated with $S$ in place of $\Lambda$.

If $A$ is a shape matrix, then we can apply the above discussion to the row and column sequences of $A$ to obtain natural direct sum decompositions

$$
\begin{aligned}
& \Lambda_{A} \phi=\sum_{a+b=|A|} \Lambda_{A}\left(\phi_{1}, \phi_{2} ; a, b\right), \\
& S_{\tilde{A}} \phi=\sum_{a+b=|\tilde{A}|} S_{\tilde{A}}\left(\phi_{1}, \phi_{2} ; a, b\right)
\end{aligned}
$$

of chain complexes. It is immediate from homogeneity that the Schur map $d_{A}(\phi)$ decomposes into a direct sum $\sum_{a+b=|A|} d_{A}\left(\phi_{1}, \phi_{2} ; a, b\right)$ where each $d_{A}\left(\phi_{1}, \phi_{2} ; a, b\right)$ is a map of complexes $\Lambda_{A}\left(\phi_{1}, \phi_{2} ; a, b\right) \rightarrow S_{\tilde{A}}\left(\phi_{1}, \phi_{2} ; a, b\right)$. We can then define $\mathbf{L}_{A}\left(\phi_{1}, \phi_{2} ; a, b\right)$ to be the image of $d_{A}\left(\phi_{1}, \phi_{2} ; a, b\right)$ and obtain the natural direct sum decomposition

$$
\mathbf{L}_{A} \phi=\sum_{a+b=|A|} \mathbf{L}_{A}\left(\phi_{1}, \phi_{2} ; a, b\right)
$$

of complexes. 
Standard basis theorem. Most of what is known about the functors $\mathbf{L}_{A}\left(\_\right)$is known in the case $A=\lambda / \mu$ is a skew partition. We will give a brief summary of those properties which will be needed in this paper, beginning with the presentation of Schur complexes by generators and relations. (See [2] for details and for references to treatments of related subjects.)

If $A$ is a shape matrix of a skew partition $\lambda / \mu$, we let $p_{i}=\lambda_{i}-\mu_{i}$ for $1 \leq i \leq l(\lambda)=s$, and $t_{i}=\mu_{i}-\mu_{i+1}+1$ for $1 \leq i \leq s-1$. For each $i \leq s-1$ and $r_{i} \geq 0$, we have a map $\square_{i}$ :

$$
\Lambda^{p_{1}} \phi \otimes \cdots \otimes \Lambda^{p_{i}+t_{i}+r_{i}} \phi \otimes \Lambda^{p_{i+1}-t_{i}-r_{i}} \phi \otimes \cdots \otimes \Lambda^{p_{s}} \phi \rightarrow \Lambda^{p_{1}} \phi \otimes \cdots \otimes \Lambda^{p_{s}} \phi
$$

defined by diagonalizing $\Lambda^{p_{i}+t_{i}+r_{i}} \phi$ into $\Lambda^{p_{i}} \phi \otimes \Lambda^{t_{i}+r_{i}} \phi$ and then multiplying $\Lambda^{t_{i}+r_{i}} \phi \otimes \Lambda^{p_{i+1}-t_{i}-r_{i}} \phi$ into $\Lambda^{p_{i+1}} \phi$. We then define the map

$\square_{\lambda / \mu}(\phi): \sum_{i, r_{i}} \Lambda^{p_{1}} \phi \otimes \cdots \otimes \Lambda^{p_{i}+t_{i}+r_{i}} \phi \otimes \Lambda^{p_{i+1}-t_{i}-r_{i}} \phi \otimes \cdots \otimes \Lambda^{p_{s}} \phi \rightarrow \Lambda^{p_{1}} \phi \otimes \cdots \otimes \Lambda^{p_{s}} \phi$

to be the sum of all the above maps $\square_{i}$ as we let $i$ range from 1 to $s-1$, and the nonnegative integer $r_{i}$ range from 0 to $\lambda_{i+1}-\mu_{i}-1$. It is shown in [2] that $d_{\lambda / \mu}(\phi) \square_{\lambda / \mu}(\phi)=0$. Hence, if we set $\overline{\mathbf{L}_{\lambda / \mu}(\phi)}=\operatorname{coker} \square_{\lambda / \mu}(\phi)$, we have a natural map

$$
\Theta_{\lambda / \mu}(\phi): \overline{\mathbf{L}_{\lambda / \mu} \phi} \rightarrow \mathbf{L}_{\lambda / \mu} \phi .
$$

Theorem 2.2 [2]. For any skew partition $\lambda / \mu$, the complex $\mathbf{L}_{\lambda / \mu} \phi$ is free in each dimension, and the morphism $\Theta_{\lambda / \mu}(\phi)$ is an isomorphism. Hence, $\mathbf{L}_{\lambda / \mu} \phi$ is a universally free complex. (By this, we mean that for a given map $\phi: G \rightarrow F$ of free $R$-modules of finite ranks the component of $\mathbf{L}_{\lambda / \mu} \phi$ in each dimension is a free $R$-module of finite rank which depends only on the ranks of $G, F$ and not on the ground ring $R$.)

As the Schur complex $\mathbf{L}_{\lambda / \mu} \phi$ is universally free, we may ask what a basis for $\mathbf{L}_{\lambda / \mu} \phi$ is? There are many ways to describe a basis for $\mathbf{L}_{\lambda / \mu} \phi$ in terms of bases $\left\{g_{1}, \ldots, g_{n}\right\}$ of $G$ and $\left\{f_{1}, \ldots, f_{m}\right\}$ of $F$, but we shall describe two of the most common ways that arise in practice.

Let $B(G, F)=\left\{g_{1}, \ldots, g_{n}, f_{1}, \ldots, f_{m}\right\}$ be the union of the two bases totally ordered by

$$
g_{1}<g_{2}<\cdots<g_{n}<f_{1}<f_{2}<\cdots<f_{m}
$$

and let $B(F, G)=\left\{f_{1}, \ldots, f_{m}, g_{1}, \ldots, g_{n}\right\}$ be the same set totally ordered by $f_{1}<f_{2}<\cdots<f_{m}<g_{1}<g_{2}<\cdots<g_{n}$. Then we call a map, $T$, from $\Delta_{\lambda / \mu}$ to $B(G, F)$ a tableau in $B(G, F)$ of shape $\lambda / \mu$. The set of all such tableaux is denoted by $\operatorname{Tab}_{\lambda / \mu}(B(G, F))$. Now the domain of $d_{\lambda / \mu}(\phi)$ is $\Lambda_{\lambda / \mu} \phi=\Lambda^{\lambda_{1}-\mu_{1}} \phi \otimes \cdots \otimes \Lambda^{\lambda_{s}-\mu_{s}} \phi$ which can be viewed either as

$$
\sum_{a_{i}+b_{i}=\lambda_{i}-\mu_{i}}\left(D_{a_{1}} G \otimes \Lambda^{b_{1}} F\right) \otimes \cdots \otimes\left(D_{a_{s}} G \otimes \Lambda^{b_{s}} F\right)
$$


or as

$$
\sum_{a_{i}+b_{i}=\lambda_{i}-\mu_{i}}\left(\Lambda^{b_{1}} F \otimes D_{a_{1}} G\right) \otimes \cdots \otimes\left(\Lambda^{b_{s}} F \otimes D_{a_{s}} G\right) .
$$

A tableau $T$ in $\operatorname{Tab}_{\lambda / \mu}(B(G, F))$ is called row-standard $\bmod G$ if in each row of the tableau $T$, the entries are increasing with repeats allowed only among the basis elements of $G . T$ is called column-standard $\bmod G$ if in each column of $T$, the entries are increasing with repeats allowed only among the basis elements of $F . T$ is called standard $\bmod G$ if it is both row-standard $\bmod G$ and column-standard $\bmod G$. We note that when $G$ has the empty base (i.e., $G=$ 0 ) the tableau $T$ may be called row-standard, column-standard, or standard in the above definitions, respectively. If $F$ has the empty base (i.e., $F=0$ ), then the tableau $T$ may be called co-row-standard, co-column-standard, or costandard in the above definitions, respectively.

If, now, we take a tableau $T \in \operatorname{Tab}_{\lambda / \mu}(B(G, F))$ which is row-standard $\bmod G$, then we may associate to such a tableau a basis element $Z_{T}$ of $\Lambda_{\lambda / \mu} \phi$ as follows: if the entries in the $i$ th row of $T$ are

$$
\underbrace{g_{i_{1}} \cdots g_{i_{1}}}_{e_{i_{1}}} \underbrace{g_{i_{2}} \cdots g_{i_{2}}}_{e_{i_{2}}} \cdots \underbrace{g_{i_{k}} \cdots g_{i_{k}}}_{e_{i_{k}}} f_{i_{1}} \cdots f_{i_{b_{i}}}
$$

(where $\sum e_{i_{j}}=a_{i}$, and $a_{i}+b_{i}=\lambda_{i}-\mu_{i}$ ), then the element

$$
Z_{T_{i}}=g_{i_{1}}^{\left(e_{i_{1}}\right)} g_{i_{2}}^{\left(e_{i_{2}}\right)} \cdots g_{i_{k}}^{\left(e_{i_{k}}\right)} \otimes f_{i_{1}} \Lambda \cdots \Lambda f_{i_{b_{i}}}
$$

is a basis element of $D_{a_{i}} G \otimes \Lambda^{b_{i}} F$ and $Z_{T}=Z_{T_{1}} \otimes \cdots \otimes Z_{T_{s}}$ is a basis element of (4). Conversely, given a basis element of $Z$ of (4), we can assign to it a tableau $T_{Z}$ in $\operatorname{Tab}_{\lambda / \mu}(B(G, F))$ which is row-standard $\bmod G$. The above discussion and definitions can be repeated with $B(F, G)$ and (5) in places of $B(G, F)$ and (4). Notice that the same conclusion holds for any order of the basis elements of $G$ and $F$, not only for the ones $B(G, F)$ and $B(F, G)$ we have chosen. Thus, from now on, when we do not specify the order of the basis elements of $G$ and $F$, we assume that we have chosen some order, once and for all, and we denote by $B(\phi)$ such a totally ordered set of the basis elements of $G$ and $F$.

Theorem 2.3 [2]. A basis for $\mathbf{L}_{\lambda / \mu} \phi$ is given by $\left\{d_{\lambda / \mu}(\phi)\left(Z_{T}\right) \mid T\right.$ is standard $\bmod G$ in $\left.\operatorname{Tab}_{\lambda / \mu}(B(\phi))\right\}$.

Remark that Theorem 2.2 and 2.3 are usually called the standard basis theorem for Schur complexes. If $T$ is standard $\bmod G$ in $\operatorname{Tab}_{\lambda / \mu}(B(\phi))$, we call $d_{\lambda / \mu}(\phi)\left(Z_{T}\right)$ a standard basis element of $\mathbf{L}_{\lambda / \mu} \phi$. If $G=0$, then we call those theorems the standard basis theorem for Schur modules. But if $F=0$, then we call those the standard basis theorem for Weyl modules.

An important consequence of the standard basis theorem is the decomposition by filtration of the Schur complex $\mathbf{L}_{A}\left(\phi_{1} \oplus \phi_{2}\right)$. Let $\phi: G \rightarrow F$ be the 
direct sum $\phi_{1} \oplus \phi_{2}$ of two maps $\phi_{i}: G_{i} \rightarrow F_{i}$, and let $A=\lambda / \mu$ be a skew partition. From (2) we have the direct sum decomposition of the chain complex $\Lambda_{A} \phi$ :

$$
\Lambda_{A} \phi=\sum \Lambda_{\sigma / \mu} \phi_{1} \otimes \Lambda_{\lambda / \sigma} \phi_{2}
$$

where the sum is over all sequences $\sigma$ such that $\mu \subseteq \sigma \subseteq \lambda$. Now for any such sequence $\gamma$ we define subcomplexes

$$
\begin{aligned}
& M_{\gamma}\left(\mathbf{L}_{A} \phi\right)=d_{A}(\phi)\left(\sum_{\sigma \geq \gamma} \Lambda_{\sigma / \mu} \phi_{1} \otimes \Lambda_{\lambda / \sigma} \phi_{2}\right), \\
& \dot{M}_{\gamma}\left(\mathbf{L}_{A} \phi\right)=d_{A}(\phi)\left(\sum_{\sigma>\gamma} \Lambda_{\sigma / \mu} \phi_{1} \otimes \Lambda_{\lambda / \sigma} \phi_{2}\right),
\end{aligned}
$$

where $d_{A}(\phi): \Lambda_{A} \phi \rightarrow S_{\vec{A}} \phi$ is the Schur map and the summations are restricted to partitions $\sigma$ satisfying $\mu \subseteq \sigma \subseteq \lambda$. The inequalities $\sigma \geq \gamma$ and $\sigma>\gamma$ refer to the lexicographic order on sequences of natural numbers. Thus $\sigma>\gamma$ means that $\sigma_{1}=\gamma_{1}, \ldots, \sigma_{i}=\gamma_{i}$ and $\sigma_{i+1}>\gamma_{i+1}$ for some $i$. Notice that if $\gamma$ is not a partition, then it is clear from (6) that $M_{\gamma}\left(\mathbf{L}_{A} \phi\right)$ equals $\dot{M}_{\gamma}\left(\mathbf{L}_{A} \phi\right)$. If $\gamma$ is a partition, it is equally clear that $\dot{M}_{\gamma}\left(\mathbf{L}_{A} \phi\right)$ equals $M_{\dot{\gamma}}\left(\mathbf{L}_{A} \phi\right)$ where $\dot{\gamma}$ denotes the smallest partition $>\gamma$ in the lexicographic order.

We have a filtration $\left\{M_{\gamma}\left(\mathbf{L}_{A} \phi\right) \mid \mu \subseteq \gamma \subseteq \lambda\right\}$ of $\mathbf{L}_{A} \phi$ by chain complexes, decreasing as a partition $\gamma$ increases in the lexicographic order. It should be observed from the straightening law [2] that the summations in (6) can be allowed to range over all sequences $\sigma, \mu \subseteq \sigma \subseteq \lambda$, satisfying $\sigma \geq \gamma$ and $\sigma>\gamma$, respectively.

Theorem 2.4 [2]. If $\gamma$ is a partition, $\mu \subseteq \gamma \subseteq \lambda$, then there is a natural isomorphism of chain complexes

$$
\mathbf{L}_{\gamma / \mu} \phi_{1} \otimes \mathbf{L}_{\lambda / \gamma} \phi_{2} \cong M_{\gamma}\left(\mathbf{L}_{A} \phi\right) / \dot{M}_{\gamma}\left(\mathbf{L}_{A} \phi\right)
$$

induced by the restriction of $d_{A}(\phi): \Lambda_{A} \phi \rightarrow \mathbf{L}_{A} \phi$ to

$$
\Lambda_{\gamma / \mu} \phi_{1} \otimes \Lambda_{\lambda / \gamma} \phi_{2} \rightarrow M_{\gamma}\left(\mathbf{L}_{A} \phi\right) \text {. }
$$

Hence, the associated graded object of the filtration $\left\{M_{\gamma}\left(\mathbf{L}_{A} \phi\right)\right\}$ is naturally isomorphic to the direct sum

$$
\sum_{\gamma} \mathbf{L}_{\gamma / \mu} \phi_{1} \otimes \mathbf{L}_{\lambda / \gamma} \phi_{2}
$$

of complexes where the sum is over all partitions $\gamma$ satisfying $\mu \subseteq \gamma \subseteq \lambda$.

From the decomposition result (3) for a direct sum, this theorem can be reformulated for convenience as follows.

Corollary 2.5. If $A$ is the shape matrix corresponding to a skew partition $\lambda / \mu$, and integers $a, b$ satisfy $a+b=|A|$, then the complex $\mathbf{L}_{A}\left(\phi_{1}, \phi_{2} ; a, b\right)$ admits a natural filtration

$$
\left\{M_{\gamma}\left(\mathbf{L}_{A}\left(\phi_{1}, \phi_{2} ; a, b\right)\right)|\mu \subseteq \gamma \subseteq \lambda,| \gamma / \mu|=a,| \lambda / \gamma \mid=b\right\}
$$


by subcomplexes so that the associated graded complex is

$$
\sum \mathbf{L}_{\gamma / \mu} \phi_{1} \otimes \mathbf{L}_{\lambda / \gamma} \phi_{2}
$$

where the sum is taken over all partitions $\gamma$ such that $\mu \subseteq \gamma \subseteq \lambda,|\gamma / \mu|=a$, and $|\lambda / \gamma|=b$.

One of the consequences of Theorem 2.4 and Corollary 2.5 is a decomposition formula for the chain module $\left(\mathbf{L}_{A} \phi\right)_{j}$ in dimension $j$ of the Schur complex $\mathbf{L}_{A} \phi$, where again $A=\lambda / \mu$ is a skew partition and $\phi: G \rightarrow F$ is a map of free $R$-modules.

Corollary 2.6 [2]. There is a natural filtration by $R$-modules of $\left(\mathbf{L}_{A} \phi\right)_{j}$ whose associated graded module is naturally isomorphic to

$$
\sum_{\gamma} L_{\gamma / \mu} F \otimes K_{\lambda / \gamma} G
$$

where the sum is over all partitions $\gamma$ satisfying $\mu \subseteq \gamma \subseteq \lambda$ and $|\lambda / \gamma|=j$.

To complete this section, we stress the fact that Theorem 2.4 and Corollary 2.5 give filtrations of complex (whenever $\phi=\phi_{1} \oplus \phi_{2}$ ), while Corollary 2.6 gives a filtration of modules (for any $\phi$ ).

\section{Pieri formulas for SCHUR COMPLEXes}

In this section, we will prove the characteristic-free versions for Schur complexes of Pieri formulas for symmetric functions (or Schubert cycles).

Over a field $R$ of characteristic zero the irreducible polynomial representations of $\mathrm{GL}(F)$ are the vector spaces $L_{\lambda} F$, where $\lambda=\left(\lambda_{1}, \ldots, \lambda_{s}\right)$ with $\lambda_{1} \leq \operatorname{dim} F$. The tensor product $L_{\lambda} F \otimes S_{p} F$ is again a representation, and since $\mathrm{GL}(F)$ is linearly reductive, we have

$$
L_{\lambda} F \otimes S_{p} F \cong \sum_{\sigma} L_{\sigma} F
$$

where $\sigma$ runs over all partitions of weight $|\lambda|+p$ containing $\lambda$ subject to the restriction that the diagram of $\sigma / \lambda$ contains at most one box in each row. Similarly, the tensor product $L_{\lambda} F \otimes \Lambda^{p} F$ decomposes into the direct sum $\sum_{\rho} L_{\rho} F$ where $\rho$ again ranges over those partitions of weight $|\lambda|+p$ containing $\lambda$, but now subject to the condition that $\rho / \lambda$ contains at most one box in each column.

In [1], these two decompositions were proved in a form that is valid over any commutative ring $R$.

A natural question, then, is whether the same decompositions hold for Schur complexes. We begin with the skew Pieri formulas.

Theorem 3.1. Let $R$ be any commutative ring, $\phi: G \rightarrow F$ any homomorphism of finitely generated free $R$-modules, and $\lambda$ any partition. Then

(a) $\mathbf{L}_{\lambda /\left(1^{p}\right)} \phi$ admits a natural filtration whose associated graded complex is isomorphic to $\sum_{\mu} \mathbf{L}_{\mu} \phi$ where the sum is taken over all partitions $\mu \subseteq \lambda$ such that $|\mu|=|\lambda|-p$ and $\lambda_{i}-1 \leq \mu_{i} \leq \lambda_{i}$ for all $i$; 
(b) $\mathbf{L}_{\lambda /(p)} \phi$ admits a natural filtration whose associated graded complex is isomorphic to $\sum_{\nu} \mathbf{L}_{\nu} \phi$ where the sum is taken over all partitions $\nu \subseteq \lambda$ such that $|\nu|=|\lambda|-p$ and $\tilde{\lambda}_{j}-1 \leq \widetilde{\nu}_{j} \leq \tilde{\lambda}_{j}$ for all $j$.

Proof. (a) Let us take $\psi: 0 \rightarrow R$ to be the zero map. Using Corollary 2.5 we know that $\mathbf{L}_{\lambda}(\psi, \phi ; p,|\lambda|-p)$, which is isomorphic to $\mathbf{L}_{\lambda}(\phi, \psi ;|\lambda|-p, p)$ has two natural filtrations which yield the following two decompositions:

$$
\begin{aligned}
& \mathbf{L}_{\lambda}(\psi, \phi ; p,|\lambda|-p) \cong \sum_{\substack{\gamma \subseteq \lambda \\
|\gamma|=p}} \mathbf{L}_{\gamma} \psi \otimes \mathbf{L}_{\lambda / \gamma} \phi \text { up to filtration, } \\
& \mathbf{L}_{\lambda}(\phi, \psi ;|\lambda|-p, p) \cong \sum_{\substack{\mu \subseteq \lambda \\
|\lambda| \mu \mid=p}} \mathbf{L}_{\mu} \phi \otimes \mathbf{L}_{\lambda / \mu} \psi \text { up to filtration. }
\end{aligned}
$$

Since $\psi: 0 \rightarrow R$ is a zero map, $\mathbf{L}_{\gamma} \psi=0$, unless $\gamma$ is the partition $\left(1^{p}\right)$, and in this case $\mathbf{L}_{\left(1^{p}\right)} \psi \cong(0 \rightarrow R \rightarrow 0)$. Therefore, we have

$$
\mathbf{L}_{\lambda}(\psi, \phi ; p,|\lambda|-p) \cong \mathbf{L}_{\lambda /\left(1^{p}\right)} \phi .
$$

On the other hand, we have $\mathbf{L}_{\lambda / \mu} \psi=0$ unless $\lambda / \mu$ contains at most one box in each row, in which case $\mathbf{L}_{\lambda / \mu} \psi \cong(0 \rightarrow R \rightarrow 0)$. Hence, we get exactly the statement (a).

(b) To see the formula (b), we will follow the same line of reasoning as that of formula (a), but we need some minor modifications. Consider a zero map $\psi: R \rightarrow 0$ (see the difference in the zero maps here and there). Using Corollary 2.5 , we know that $\mathbf{L}_{\lambda}(\psi, \phi ; p,|\lambda|-p)$, which is isomorphic to $\mathbf{L}_{\lambda}(\phi, \psi ;|\lambda|-p, p)$, has two natural filtrations which yield the following two decompositions:

$$
\begin{aligned}
& \mathbf{L}_{\lambda}(\psi, \phi ; p,|\lambda|-p) \cong \sum_{\substack{\gamma \subseteq \lambda \\
|\gamma|=p}} \mathbf{L}_{\gamma} \psi \otimes \mathbf{L}_{\lambda / \gamma} \phi \text { up to filtration, } \\
& \mathbf{L}_{\lambda}(\phi, \psi ;|\lambda|-p, p) \cong \sum_{\substack{\nu \subseteq \lambda \\
|\lambda / \nu|=p}} \mathbf{L}_{\nu} \phi \otimes \mathbf{L}_{\lambda / \nu} \psi \text { up to filtration. }
\end{aligned}
$$

Since $\psi: R \rightarrow 0$ is a zero map, $\mathbf{L}_{\gamma} \psi=0$ unless $\gamma$ is the partition $(p)$, and in this case $\mathbf{L}_{(p)} \psi \cong(0 \rightarrow R \rightarrow 0)$. Therefore, we have $\mathbf{L}_{\lambda}(\psi, \phi ; p,|\lambda|-p) \cong$ $\mathbf{L}_{\lambda /(p)} \phi$. On the other hand, we have $\mathbf{L}_{\lambda / \nu} \psi=0$ unless $\lambda / \nu$ contains at most one box in each column, in which case $\mathbf{L}_{\lambda / \nu} \psi \cong(0 \rightarrow R \rightarrow 0)$. Thus, formula (b) now follows from the above discussion.

As special cases of Theorem 3.1, we obtain the Pieri formulas for Schur complexes.

Theorem 3.2. Let $R$ be any commutative ring, $\phi: G \rightarrow F$ any homomorphism of finitely generated free modules, and $\lambda$ any partition. Then 
(a) $\mathbf{L}_{\lambda} \phi \otimes S_{p} \phi$ admits a natural filtration whose associated graded complex is isomorphic to $\sum_{\sigma} \mathbf{L}_{\sigma} \phi$ where the sum is taken over all partitions $\sigma$ such that $|\sigma|=|\lambda|+p$ and $\lambda_{i} \leq \sigma_{i} \leq \lambda_{i}+1$ for all $i$;

(b) $\mathbf{L}_{\lambda} \phi \otimes \Lambda^{p} \phi$ admits a natural filtration whose associated graded complex is isomorphic to $\sum_{\rho} \mathbf{L}_{\rho} \phi$ where the sum is taken over all partitions $\rho$ such that $|\rho|=|\lambda|+p$ and $\tilde{\lambda}_{j} \leq \widetilde{\rho}_{j} \leq \tilde{\lambda}_{j}+1$ for all $j$.

Proof. (a) If we let $\lambda=\left(\lambda_{1}, \ldots, \lambda_{s}\right)$ be a partition with $\lambda_{s} \neq 0$ and

$$
\lambda^{\prime}=(\lambda_{1}+1, \ldots, \lambda_{s}+1, \underbrace{1, \ldots, 1}_{p})
$$

a partition formed by adjoining $s+p$ ones to $\lambda$, then it follows from the definition of the Schur complex that $\mathbf{L}_{\lambda} \phi \otimes S_{p} \phi \cong \mathbf{L}_{\lambda^{\prime} /\left(1^{s}\right)} \phi$. A diagram of $\lambda^{\prime} /\left(1^{s}\right)$ is pictured below:

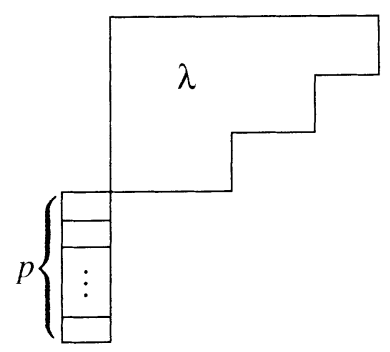

If we now apply formula (a) of Theorem 3.11 , we immediately recover formula (a).

(b) As regards formula (b), we take $\lambda^{\prime \prime}$ to be the partition $\left(\lambda_{1}+p, \lambda_{1}, \ldots, \lambda_{s}\right)$ and note that $\mathbf{L}_{\lambda} \phi \otimes \lambda^{p} \phi \cong \mathbf{L}_{\lambda^{\prime \prime} /\left(\lambda_{1}\right)} \phi$. We then apply formula (b) of Theorem 3.1 to $\mathbf{L}_{\lambda^{\prime \prime} /\left(\lambda_{1}\right)} \phi$ and obtain formula (b).

Recalling that if $\phi: 0 \rightarrow F$ (that is, $G=0$ ) then $\mathbf{L}_{\lambda / \mu} \phi$ is $L_{\lambda / \mu} F$ in dimension zero, and if $\phi: G \rightarrow 0$ (that is, $F=0$ ) then $\mathbf{L}_{\lambda / \mu} \phi$ is $K_{\lambda / \mu} G$ in dimension $|\lambda / \mu|$ (the number of boxes in the skew shape), we obtain the following results.

Corollary 3.3. Let $R$ be any commutative ring, and let $F$ denote any finitely generated free $R$-module. Then one has the isomorphisms (up to filtrations, obviously):

(a) $L_{\lambda /\left(1^{p}\right)} F \cong \sum_{\mu} L_{\mu} F$, where $\mu$ runs over all partitions such that $\mu \subseteq \lambda$, $|\mu|=|\lambda|-p$, and $\lambda_{i}-1 \leq \mu_{i} \leq \lambda_{i}$ for all $i$;

(b) $L_{\lambda /(p)} F \cong \sum_{\nu} L_{\nu} F$, where $\nu$ runs over all partitions such that $\nu \subseteq \lambda$, $|\nu|=|\lambda|-p$, and $\tilde{\lambda}_{j}-1 \leq \widetilde{\nu}_{j} \leq \tilde{\lambda}_{j}$ for all $j$;

(c) $K_{\lambda /\left(1^{p}\right)} F \cong \sum_{\mu} K_{\mu} F$, where $\mu$ runs over all partitions such that $\mu \subseteq \lambda$, $|\mu|=|\lambda|-p$, and $\lambda_{i}-1 \leq \mu_{i} \leq \lambda_{i}$ for all $i$;

(d) $K_{\lambda /(p)} F \cong \sum_{\nu} F$, where $\nu$ runs over all partitions such that $\nu \subseteq \lambda$, $|\nu|=|\lambda|-p$, and $\tilde{\lambda}_{j}-1 \leq \widetilde{\nu}_{j} \leq \tilde{\lambda}_{j}$ for all $j$. 
Corollary 3.4. Let $R$ be any commutative ring, and let $F$ denote any finitely generated free $R$-module. Then one has the isomorphisms (up to filtration, obviously):

(a) $L_{\lambda} F \otimes S_{p} F \cong \sum_{\sigma} L_{\sigma} F$, where $\sigma$ runs over all partitions such that $\sigma \supseteq \lambda$, $|\sigma|=|\lambda|+p$, and $\lambda_{i} \leq \sigma_{i} \leq \lambda_{i}+1$ for all $i$;

(b) $L_{\lambda} F \otimes \Lambda^{p} F \cong \sum_{\rho} L_{\rho} F$, where $\rho$ runs over all partitions such that $\rho \supseteq \lambda$, $|\rho|=|\lambda|+p$, and $\tilde{\lambda}_{j} \leq \tilde{\rho}_{j} \leq \tilde{\lambda}_{j}+1$ for all $j$;

(c) $K_{\lambda} F \otimes \Lambda^{p} F \cong \sum_{\sigma} K_{\sigma} F$, where $\sigma$ runs over all partitions such that $\sigma \supseteq \lambda$, $|\sigma|=|\lambda|+p$, and $\lambda_{i} \leq \sigma_{i} \leq \lambda_{i}+1$ for all $i$;

(d) $K_{\lambda} F \otimes D_{p} G \cong \sum_{\rho} K_{\rho} F$, where $\rho$ runs over all partitions such that $\rho \supseteq \lambda$, $|\rho|=|\lambda|+p$, and $\tilde{\lambda}_{j} \leq \widetilde{\rho}_{j} \leq \tilde{\lambda}_{j}+1$ for all $j$.

\section{LITTLEWOOD-RICHARDSON RULES}

In this section we will provide useful descriptions of Littlewood-Richardson coefficients. Let us first review some preliminary notions in Combinatorics. A finite sequence $\mathbf{a}=\left(a_{1}, \ldots, a_{n}\right)$ of positive integers is said to be a lattice permutation, if for each $k=1, \ldots, n$, the number of times $i$ appears in the subsequences $\left(a_{1}, \ldots, a_{k}\right)$ is not less than the number of times $i+1$ appears in there, for every positive integer $i$. E.g., $(1,1,2,2,1,2,3)$ is a lattice permutation; $(1,1,2,2,2,1,3)$ is not a lattice permutation. If $\mathbf{a}=\left(a_{1}, \ldots, a_{n}\right)$ is any sequence of positive integers, its content is defined to be the sequence $\nu=\left(\nu_{1}, \nu_{2}, \ldots\right)$, where $\nu_{i}$ is the number of times $i$ appears in a. It follows that $\mathbf{a}$ is a lattice permutation if and only if the content of $\left(a_{1}, \ldots, a_{k}\right)$ is a partition for each $k=1, \ldots, n$. More generally, a finite sequence $\left(x_{i_{1}}, \ldots, x_{i_{m}}\right)$ from an ordered set $B=\left\{x_{1} \leq \cdots \leq x_{n}\right\}$ is called a lattice permutation if $\left(i_{1}, \ldots, i_{m}\right)$ forms a lattice permutation of numbers.

Lemma 4.1 [2]. There is a bijection between the set of lattice permutations of content $\nu$ and the set of standard tableaux of shape $\nu$ with distinct entries from the set $\{1,2, \ldots, n\}$, where $n$ is the weight of $\nu$.

Proof. To see this let $T$ be row-standard tableau of shape $\nu$ with distinct entries $1, \ldots, n$. Each $i \in\{1, \ldots, n\}$ appears as an entry $T(j, k)$. Let $a_{i}=j$ and define $\alpha(T)$ to be the sequence $\left(a_{1}, \ldots, a_{n}\right)$ of content $\nu$. Conversely, if $\mathbf{a}=\left(a_{1}, \ldots, a_{n}\right)$ is a sequence of content $\nu$, let $\left(a_{k_{1}}, \ldots, a_{k_{\nu_{i}}}\right)$ be the subsequence of a with entries equal to $i$. Define $\beta(\mathbf{a})$ to be the row-standard tableau of shape $\nu$ given by $\beta(\mathbf{a})(i, j)=k_{j}$. It is clear that $\alpha$ and $\beta$ are inverses. An easy induction argument on $n$ shows that $\beta$ takes lattice permutations to standard tableaux and that $\alpha$ does the reverse, giving the desired bijection.

As an illustration, $\beta$ takes the lattice permutation $(1,1,2,1,3,2)$ to the following tableau. 


\begin{tabular}{|l|l|l|}
\hline 1 & 2 & 4 \\
\hline 3 & 6 & \\
\cline { 1 - 2 } 5 & \multicolumn{2}{|c}{} \\
\cline { 1 - 2 } &
\end{tabular}

Suppose that $T$ is a tableau of shape $\nu$. Then its transpose $\widetilde{T}$ is the tableau of shape $\widetilde{\nu}$ given by $\widetilde{T}(i, j)=T(j, i)$. It is clear that $\widetilde{\widetilde{T}}=T$ and if $T$ is a standard tableau with distinct entries, then so is $\widetilde{T}$. Now let $\mathbf{a}=\left(a_{1}, \ldots, a_{n}\right)$ be a lattice permutation of content $\nu$. We define its transpose $\widetilde{\mathbf{a}}$ to be the lattice permutation $\alpha(\widetilde{\beta}(\mathbf{a}))$ of content $\widetilde{\nu}$, so that $\widetilde{\mathbf{a}}=\mathbf{a}$. It is easy to see that $\widetilde{\mathbf{a}}=\left(\widetilde{a}_{1}, \ldots, \widetilde{a}_{n}\right)$, where $\widetilde{a}_{i}$ is the number of $a_{k}$ such that $a_{k}=a_{i}$ and $k \leq i$. Furthermore, we define the content of a tableau $T$ in $\operatorname{Tab}_{\lambda / \nu}(\{1,2, \ldots, n\})$ to be the sequence in $\mathbf{N}^{\infty}$ whose $i$ th term is the number of times the element $i$ appears as an entry in $T$.

Now let $T$ be a standard tableau in $\operatorname{Tab}_{\lambda / \nu}(\{1,2, \ldots, n\})$. We define two sequences Ass $T$ and $\mathscr{A} s s T$ associated with $T$ as follows:

- Ass $T$ is the sequence of $T$ formed by listing the entries of $T$ from bottom to top in each column, starting with the left-most column and continuing to the right;

- $\mathscr{A} S S T$ is the sequence of $T$ formed by listing the entries of $T$ from left to right in each row, starting with the bottom row and continuing to the top.

Definition 4.2. The Littlewood-Richardson coefficient (or LR-coefficient, for short) $C_{\mu \nu}^{\lambda}$ is the number of standard tableaux $T$ in $\operatorname{Tab}_{\lambda / \nu}\left(\left\{1, \ldots, \mu_{1}\right\}\right)$ of content $\widetilde{\mu}$ such that Ass $T$ is a lattice permutation.

It is easy to see that $C_{\mu \nu}^{\lambda}=C_{\nu \mu}^{\lambda}$, and $C_{\mu \nu}^{\lambda}=0$ unless $|\lambda|=|\mu|+|\nu|$ and $\mu, \nu \subseteq \lambda$. A tableau $T$ is a Littlewood-Richardson ("LR", for short) tableau if Ass $T$ is a lattice permutation. Thus $C_{\mu \nu}^{\lambda}$ is the number of standard LRtableaux of content $\widetilde{\mu}$ in $\operatorname{Tab}_{\lambda / \nu}\left(\left\{1,2, \ldots, \mu_{1}\right\}\right)$. Now we will prove that Ass $T$ may be replaced by $\mathscr{A} s S T$ in the above definition. This allows us a little bit more freedom for computing the coefficients $C_{\mu \nu}^{\lambda}$ in practice. For the convenience of simplicity, we consider all the tableaux in the following as those with values in $\{1,2, \ldots, n\}$ for a suitable integer $n$.

Proposition 4.3. Given a pair of partitions $\nu \subseteq \lambda$, let $T$ be a standard $L R$ tableau of shape $\lambda / \nu$ that has more than one row. If $T_{1}$ is the tableau obtained by erasing the top row of $T$, then Ass $T_{1}$ is a lattice permutation.

Proof. If we let Ass $T_{1}=\left(a_{1}, a_{2}, \ldots, a_{n}\right)$, then it is clearly enough to show that the content of $\left(a_{1}, \ldots, a_{k}\right)$ is a partition for each $k=1, \ldots, n$. From the proof of Lemma 4.1, $\beta$ (Ass $T$ ) is a tableau whose shape is the content of $T$, and the entries in the top row of $T$ correspond to the outside boxes in the rows of the diagram of $\beta$ (Ass $T$ ). If we let $\mathbf{a}^{\prime}$ to be a subsequence listed from 
$a_{1}$ up to $a_{k}$ in Ass $T$ (note that $a_{1}$ and $a_{k}$ are not in the top row of $T$ ), the content of $\mathbf{a}^{\prime}$ is a partition because Ass $T$ is a lattice permutation. Notice that the sequence $\left(a_{1}, \ldots, a_{k}\right)$ can be obtained from $\mathbf{a}^{\prime}$ by erasing all the entries in the top row of $T$. Now observe that the diagram obtained by erasing an extremal box in the diagram whose shape is a partition has again the shape of a partition. (A box is extremal if it is an outside corner, i.e., if it has no neighbor to its right or below it.) If we let

$$
b_{1} \cdots b_{l}
$$

be the top row of $T$ and the entries $b_{1}<b_{2}<\cdots<b_{i}$ are involved in the sequence $\mathbf{a}^{\prime}$, then the box in $\beta\left(\mathbf{a}^{\prime}\right)$ corresponding to $b_{i}$ is extremal. Thus, the diagram obtained by erasing that extremal box in $\beta\left(\mathbf{a}^{\prime}\right)$ corresponding to $b_{i}$ has the shape of a partition. Using the same argument as above, we next keep erasing the boxes in $\beta\left(\mathbf{a}^{\prime}\right)$ corresponding to $b_{i-1}, \ldots, b_{1}$, one after another, to obtain the sequence of the diagrams whose shapes are partitions. This shows that the diagram of $\beta\left(\left(a_{1}, \ldots, a_{k}\right)\right)$ has the shape of a partition. It then follows from Lemma 4.1 that the content of $\left(a_{1}, \ldots, a_{k}\right)$ is a partition.

Theorem 4.4. Given a pair of partitions $\nu \subseteq \lambda$, let $T$ be a standard LR-tableau of shape $\lambda / \nu$. Then $\mathscr{A} s s T$ is a lattice permutation.

Proof. We shall proceed by induction on the number $r(\lambda / \nu)$ of rows of the diagram $\Delta_{\lambda / \nu}$, observing that $\mathscr{A} s s T=$ Ass $T$ is a lattice permutation in the trivial case $r(\lambda / \nu)=1$. Assuming the theorem is true for all such $T$ 's with the number of rows $\leq r(\lambda / \nu)-1$, let us prove it for a standard LR-tableau $T$ with $r(\lambda / \nu)$ rows. From Proposition 4.3, Ass $T_{1}$ is a lattice permutation and so $\mathscr{A}$ ss $T_{1}:=\left(a_{1}, \ldots, a_{n}\right)$ is a lattice permutation by our induction hypothesis. Using the same notation as in Proposition 4.3, we have

$$
\mathscr{A} s s T=\left(a_{1}, \ldots, a_{n}, b_{1}, \ldots, b_{l}\right) \text {. }
$$

Therefore, it is clearly enough to show that the content of $\left(a_{1}, \ldots, a_{n}\right.$, $\left.b_{1}, \ldots, b_{i}\right)$ is a partition for each $i=1, \ldots, l$. As $b_{1}<\cdots<b_{l}$ are the entries corresponding to the outside boxes in the rows of the diagram of $\beta$ (Ass $T$ ), we erase the boxes of $\beta$ (Ass $T$ ) corresponding to the entries $b_{l}, b_{l-1}, \ldots, b_{i+1}$ (starting from $b_{l}$ up to $b_{i+1}$, one after another), and then we get the diagram of $\beta\left(\left(a_{1}, \ldots, a_{n}, b_{1}, \ldots, b_{i}\right)\right)$. From the proof of Proposition 4.3, the shape of the diagram of $\beta\left(\left(a_{1}, \ldots, a_{n}, b_{1}, \ldots, b_{i}\right)\right)$ is a partition, so that the content of $\left(a_{1}, \ldots, a_{n}, b_{1}, \ldots, b_{i}\right)$ is a partition. Hence, $\mathscr{A} s s T$ is a lattice permutation.

Next, we claim that if $T$ is any standard tableau and $\mathscr{A} s S T$ is a lattice permutation then Ass $T$ is also a lattice permutation. Using the same methods given in the proofs of Proposition 4.3 and Theorem 4.4 with the column arguments in place of the row arguments, we obtain:

Proposition 4.5. Given a pair of partitions $\nu \subseteq \lambda$, let $T$ be a standard tableau of shape $\lambda / \nu$ that has more than one column, and let $\mathscr{A} s S T$ be a lattice per- 
mutation. If $T^{1}$ is the tableau obtained by erasing the right-most column of $T$, then $\mathscr{A} s s T^{1}$ is a lattice permutation.

Theorem 4.6. Given a pair of partitions $\nu \subseteq \lambda$, let $T$ be a standard tableau of shape $\lambda / \nu$, and let $\mathscr{A} s s T$ be a lattice permutation. Then Ass $T$ is a lattice permutation.

On the basis of the above theorems, we state the Littlewood-Richardson coefficients in the following alternative form.

Corollary 4.7. The LR coefficient $C_{\mu \nu}^{\lambda}$ is the number of standard tableaux $T$ in $\operatorname{Tab}_{\lambda / \nu}\left(\left\{1, \ldots, \mu_{1}\right\}\right)$ of content $\widetilde{\mu}$ such that $\mathscr{A} s s T$ is a lattice permutation.

To complete this section, we give a few other rules for the LR coefficients. We have already noticed in $\S 2$ the dual nature of $d_{\lambda / \mu}\left({ }_{-}\right)$and $d_{\lambda / \mu}^{\prime}\left({ }_{-}\right)$. It is proved as a consequence of the universal freeness of (co)Schur functors in [2] that $\left(L_{\lambda / \mu} F\right)^{*} \cong K_{\tilde{\lambda} / \tilde{\mu}}\left(F^{*}\right)$. Working with a field $R$ of characteristic zero, we know from the Littlewood-Richardson rule $[2,3]$ that

$$
\begin{aligned}
& L_{\lambda / \mu} F \cong \sum_{\mu \subseteq \nu \subseteq \lambda} C_{\mu \nu}^{\lambda} L_{\nu} F \text { and } \\
& K_{\tilde{\lambda} / \tilde{\mu}} F \cong \sum_{\tilde{\mu} \subseteq \tilde{\nu} \subseteq \tilde{\lambda}} C_{\tilde{\mu} \tilde{\nu}}^{\tilde{\lambda}} K_{\tilde{\nu}} F .
\end{aligned}
$$

Since $\operatorname{GL}(F)$ is linear reductive, the $\operatorname{GL}(F)$-modules $L_{\nu} F$ and $K_{\tilde{\nu}} F$ are irreducible and isomorphic, so we have $C_{\mu \nu}^{\lambda}=C_{\tilde{\mu} \tilde{\nu}}^{\tilde{\lambda}}$. Hence, we have the following result.

Proposition 4.8. The $L R$ coefficient $C_{\mu \nu}^{\lambda}$ is the number of standard tableaux $T$ in $\operatorname{Tab}_{\tilde{\lambda} / \tilde{\nu}}\left(\left\{1, \ldots, \tilde{\mu}_{1}\right\}\right)$ of content $\mu$ such that Ass $T$ (or equivalently, $\mathscr{A} s s T$ ) is a lattice permutation.

Finally, we transpose all standard tableaux in the above discussion to reformulate the results in terms of costandard tableaux.

Corollary 4.9. The $L R$ coefficient $C_{\mu \nu}^{\lambda}$ is the number of costandard tableaux $T$ in $\operatorname{Tab}_{\tilde{\lambda} / \hat{\nu}}\left(\left\{1, \ldots, \mu_{1}\right\}\right)$ of content $\widetilde{\mu}$ such that the sequence associated to $T$ (formed by listing the entries of $T$ from top to bottom in each column, starting with the right-most column) is a lattice permutation.

Corollary 4.10. The $L R$ coefficient $C_{\mu \nu}^{\lambda}$ is the number of costandard tableaux $T$ in $\operatorname{Tab}_{\lambda / \nu}\left(\left\{1, \ldots, \widetilde{\mu}_{1}\right\}\right)$ of content $\mu$ such that the sequence associated to $T$ (formed by listing the entries of $T$ from top to bottom in each column, starting with the right-most column) is a lattice permutation.

Acknowledgment. The author is grateful to Professor D. A. Buchsbaum, whose guidance and encouragement were invaluable in the preparation of this work. He would also like to thank the referee for some helpful comments. 


\section{REFERENCES}

1. K. Akin and D. A. Buchsbaum, Characteristic-free representation theory of the general linear group, Adv. in Math. 58 (1985), 149-200.

2. K. Akin, D. A. Buchsbaum, and J. Weyman, Schur functors and Schur complexes, Adv. in Math. 44 (1982), 207-278.

3. M. Barnabei and A. Brini, The Littewood-Richardson rule for Coschur modules, Adv. in Math. 67 (1988), 143-173.

4. C. DeConcini, D. Eisenbud, and C. Procesi, Young diagrams and determinantal varieties, Invent. Math. 56 (1980), 129-165.

5. J. A. Green, Polynomial representations of $\mathrm{GL}_{n}$, Lecture Notes in Math. vol. 830, SpringerVerlag, Berlin, 1980.

6. H. J. Ko, Thesis, Brandeis University, 1987.

7. I. G. Macdonald, Symmetric functions and Hall polynomials, Oxford Univ. Press (Clarendon), Oxford, 1979.

8. H. A. Nielsen, Tensor functors of complexes, Aarhus University Preprint Series No. 15, 1978.

Department of Mathematical Sciences, Purdue University Calumet, Hammond, IndiANA 46323

Current address: Department of Mathematics, Yonsei University, Seoul 120-749, Korea 